\title{
Analytical Analysis of Steel Reinforced Concrete Transfer Beam
}

\author{
Fan-shen Meng', a \\ ${ }^{1}$ School of Civil Engineering of Luoyang Institute of Science and Technology, Luoyang 471023, \\ China \\ aMfs2025@126.com
}

Keywords: Steel reinforced concrete; Transfer beam; Shear wall; Analytical analysis; The finite element

Abstract. In this paper, using the method of stress function analysis of steel reinforced concrete transfer beam and upper shear wall mechanical characteristics and deformation law of interaction, its stress distribution field has been given; Nonlinear finite element calculation and combined with some engineering data comparison, the result is consistent. Components as eccentric tension, the transfer beam and shear wall must be considered to participate in the work. In the shear wall and transformation tie-beam shear and vertical compressive stress should be considered, and considered as a combination.

\section{Introduction}

Structural transformation layer is the important components of high-rise building structure. Using the steel reinforced concrete ( SRC) transfer beam structure system can improve the bearing capacity and stiffness; improve the performance of the box beam's work; improve and make the structure light and beautiful, and reduce the cost. When the transfer beam design by the rules in [1] according to the bearing capacity of single beam calculation equivalent, its result is often large difference. Studies have shown that [2 5]. SRC transfer beam and upper shear wall between work together. Although this kind of structure has used in certain application and research, but also was quite in lack of theory research. In this paper stress function method is used for SRC transfer beam and upper shear wall joint action between the analysis of the stress and deformation law. It is concluded that the stress distribution field of the structure, and combined with a project example has carried on the finite element calculation. This study can provide the research and design of the structure of SRC transfer beam theory basis.

\section{Mechanical Model}

According to the steel framed structures and mechanical characteristics of SRC transfer beam, adopt the following basic assumptions. Its cross section is shown in figure 1 .

The structure is in elastic state; Section steel, steel section have been into concrete section, according to gay homogeneous material processing.

In its own plane, floor stiffness is infinite.

The shear wall along the cross section of the transfer beam constraint direction evenly has applied to transfer beam with shear wall contact area, regardless of the wall, beam contact local stress concentration.

It was selected one common structure: by the upper three layers shear walls, SRC transfer beam, 1 layer of frame column frame shear wall structure as the research object, as shown in figure 2. Based surface according to the fixed end frame column must be considered.

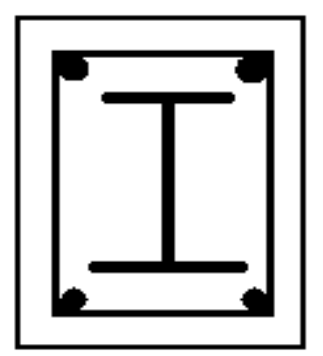

Fig1.SRC section

\section{Stress Function Method to Solve}

Polynomial analytic solution of shear wall:

As a result of the structure symmetry, it will take half of shear wall as the research object, regardless of weight. In shear wall axis upper vertex as the origin of coordinates, to the right as the $\mathrm{x}$ axis, down to 
y axis, a coordinate system was established. According to the flat plate stress inverse solution will solve the problem of elastic mechanics.

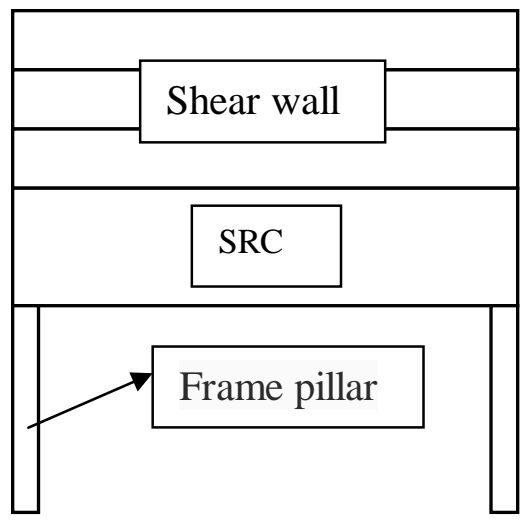

Fig. 2 The model of single SRC transfer beam

It takes stress function for

$$
U=b\left(x-\frac{L}{2}\right)^{4} y+2 d\left(x-\frac{L}{2}\right)^{2} y^{3}-\frac{1}{5}(b+d) y^{5}+e\left(x-\frac{L}{2}\right)^{2}+f\left(x-\frac{L}{2}\right) y
$$

Here $b, d, e, f$ are the undetermined constants, $\mathrm{L}$ is beam span length calculation, $Q$ is the upper structure under uniform pressure. The $U$ meets the coordinate equation, and have

$$
\begin{gathered}
\sigma_{x}=\frac{\partial^{2} U}{\partial y^{2}}=12 d\left(x-\frac{L}{2}\right)^{2} y-4(b+d) y^{3} \\
\sigma_{y}=\frac{\partial^{2} U}{\partial x^{2}}=12 b\left(x-\frac{L}{2}\right)^{2} y+4 d y^{3}+2 e \\
\tau_{x y}=-\frac{\partial^{2} U}{\partial x \partial y}=-4 b\left(x-\frac{L}{2}\right)^{3}-12 d\left(x-\frac{L}{2}\right) y^{2}-f
\end{gathered}
$$

According to the boundary conditions and the symmetry of structure and load, will get $e=-\frac{Q}{2}$, $b=-d, f=\frac{1}{2} b L^{3}-6 b L h_{1}{ }^{2} ;$ Which $h_{1}$ is the height of the structure of shear wall, at the same time can also be calculated; $b=\frac{4 Q}{h_{1}\left(8 h_{1}^{2}+L^{2}\right)}$. The stress field of shear wall will be gotten.

The triangle series solution to solve the transfer beam:

Because in below there is only side column, the transfer beam is constrained by discontinuity, the trigonometric series method is adopted; according to the rectangular analytical solution, regardless of weight. To convert the beam at the left upper vertex to the origin, to the right as the $\mathrm{x}$ direction, down for the y direction, a coordinate system was established.

It takes stress function for $U=X(x) Y(y)$, The general solution of coordinate equation is

$$
U=\left(K_{1} \cos \lambda x+K_{2} \sin \lambda x\right)(A \operatorname{ch} \lambda y+B \operatorname{sh} \lambda y+C y \operatorname{ch} \lambda y+D y \operatorname{sh} \lambda y)
$$

Here $K_{1}, K_{2}, A, B, C, D, \lambda$ are undetermined constants, $b_{0}$ is the equivalent cross section for the beam width.

The boundary conditions are $\left.\sigma_{x}\right|_{x=0}=0,\left.\sigma_{x}\right|_{x=L}=0 ; x=0, L, \sigma_{x}=\frac{\partial^{2} U}{\partial y^{2}}=0$;

$$
\text { And here: } \quad K_{1}=0, \lambda_{m}=\frac{m \pi}{L}, \quad m=1,2,3 \mathrm{~L}
$$


By $K_{2}=1$, In (1) type, and get the following series expression

$$
\begin{gathered}
U=\sum_{m=1}^{\infty} \sin \frac{m \pi x}{L}\left(A_{m} \operatorname{ch} \frac{m \pi y}{L}+B_{m} \operatorname{sh} \frac{m \pi y}{L}+C_{m} y \operatorname{ch} \frac{m \pi y}{L}+D_{m} y \operatorname{sh} \frac{m \pi y}{L}\right) \\
\sigma_{x}=\frac{\partial^{2} U}{\partial y^{2}}=\sum_{m=1}^{\infty} \frac{m^{2} \pi^{2}}{L^{2}} \sin \frac{m \pi x}{L}\left(A_{m} \operatorname{ch} \frac{m \pi y}{L}+B_{m} \operatorname{sh} \frac{m \pi y}{L}+2 \frac{L C_{m}}{m \pi} \operatorname{sh} \frac{m \pi y}{L}+\right. \\
\left.2 \frac{L D_{m}}{m \pi} \operatorname{ch} \frac{m \pi y}{L}+C_{m} y \operatorname{ch} \frac{m \pi y}{L}+D_{m} y \operatorname{sh} \frac{m \pi y}{L}\right) \\
\sigma_{y}=\frac{\partial^{2} U}{\partial x^{2}}=-\sum_{m=1}^{\infty} \frac{m^{2} \pi^{2}}{L^{2}} \sin \frac{m \pi x}{L}\left(A_{m} \operatorname{ch} \frac{m \pi y}{L}+B_{m} \frac{m \pi y}{L}+C_{m} y \operatorname{ch} \frac{m \pi y}{L}\right. \\
\left.+D_{x y} y \operatorname{sh} \frac{m \pi y}{L}\right) \\
\frac{\partial^{2} U}{\partial x \partial y}=-\sum_{m=1}^{\infty} \frac{m^{2} \pi^{2}}{L^{2}} \cos \frac{m \pi x}{L}\left(A_{m} \operatorname{sh} \frac{m \pi y}{L}+B_{m} \operatorname{ch} \frac{m \pi y}{L}+\frac{L C_{m}}{m \pi} \operatorname{ch} \frac{m \pi y}{L}\right. \\
\left.+\frac{L D_{m}}{m \pi} \operatorname{sh} \frac{m \pi y}{L}+C_{m} y s h \frac{m \pi y}{L ;}+D_{m} y c h \frac{m \pi y}{L}\right)
\end{gathered}
$$

Here $h$ is beam height. By the boundary conditions of available, It will be gotten:

$$
\begin{gathered}
A_{m}=\frac{b_{1}}{b_{0}} \times \frac{2 L}{m^{2} \pi^{2}} \int_{0}^{L}\left[\left.\sigma_{y}^{\prime}\right|_{y^{\prime}=h_{1}}\right] \sin \frac{m \pi x}{L} d x \\
B_{m}+\frac{L C_{m}}{m \pi}=\frac{b_{1}}{b_{0}} \times \frac{2 L}{m^{2} \pi^{2}} \int_{0}^{L}\left[\left.\tau_{x y}^{\prime}\right|_{y^{\prime}=h_{1}}\right] \cos \frac{m \pi x}{L} d x \\
A_{m} \operatorname{ch} \frac{m \pi h}{L}+B_{m} \operatorname{sh} \frac{m \pi h}{L}+C_{m} h c h \frac{m \pi h}{L}+D_{m} h \operatorname{sh} \frac{m \pi h}{L}=-\frac{2 L}{m^{2} \pi^{2}} \int_{0}^{L} \varphi_{1}(x) \sin \frac{m \pi x}{L} d x \\
A_{m} \operatorname{sh} \frac{m \pi h}{L}+B_{m} \operatorname{ch} \frac{m \pi h}{L}+C_{m} \frac{L}{m \pi} \operatorname{ch} \frac{m \pi h}{L}+C_{m} h \operatorname{sh} \frac{m \pi h}{L}+D_{m} \frac{L}{m \pi} \operatorname{sh} \frac{m \pi h}{L} \\
+D_{m} h \operatorname{ch} \frac{m \pi h}{L}=-\frac{2 L}{m^{2} \pi^{2}} \int_{0}^{L} \varphi_{2}(x) \cos \frac{m \pi x}{L} d x
\end{gathered}
$$

Among them, $\varphi_{1}(x)$ is the beam under compressive stress, $\varphi_{2}(x)$ is the shear stress distribution function respectively. By (2) (5), the $A_{m}, B_{m}, C_{m}, D_{m}$ can be solved, generation of response force formula, the analytical solution of the transfer beam will be given.

If $m=1,3,5 \mathrm{~L}$, the stress distribution to conform to the actual situation and assumption. Here $m=1$, and $A_{1}, B_{1}, C_{1}, D_{1}$ are work out; generation of response force component of the formula, the analytic solution of the transfer beam can be gotten.

\section{Nonlinear finite element calculation results are compared with analytical results}

On a certain engineering example was calculated by use of ANSYS software. The software function is stronger, it can undertake the structure of the nonlinear finite element calculation. It is a commonly used finite element calculation software. The transfer beam, beam end section on surface and in the 
beam cross section and the beam middle span deflection comparison calculations have been gotten. Its results were compared with analytical solutions in this paper. The corresponding calculation results listed in table 1.

Table. 1 The results of elastic mechanics analysis and nonlinear finite element method

\begin{tabular}{cccc}
\hline & $\begin{array}{r}\text { Bending moment }\left[10^{4} \mathrm{kN} \cdot\right. \\
\mathrm{m}]\end{array}$ & $\begin{array}{c}\text { shear force } \\
{\left[10^{3} \mathrm{KN}\right]}\end{array}$ & $\begin{array}{c}\text { deflection } \\
{[\mathrm{mm}]}\end{array}$ \\
\hline S A & 2.9499 & 2.6754 & -- \\
S B & 1.0864 & 4.7647 & -- \\
S C & 1.1677 & 0 & 3.60 \\
F A & 2.7217 & 1.7922 & - \\
F B & 0.9950 & 3.5943 & -- \\
F C & 0.9184 & 0.0001 & 2.60
\end{tabular}

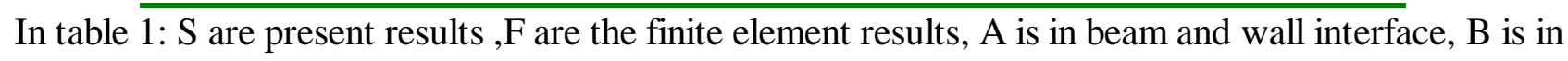
the beam end section, $\mathrm{C}$ is in the beam cross section.

It can be seen from table 1: the project under the actual working condition, the SRC transfer beam and upper shear wall of the combination of the stress distribution and deflection was consistent with the analysis results; But the comparative content is slightly bigger than non-linear finite element results of the numerical result.

\section{Conclusion}

In this paper, analytical analysis and finite element calculation is approximate results. Shear wall with conversion beam as a whole are bending deformation. Transfer beam is in the overall bending tensile flange, appeared the axial tension. Upper shear wall of the vertical load to the transfer beam, a significant portion of the load in the form of oblique load act on the end of the transfer beam. Therefore, should focus on eccentric tension across the transfer beam, shear walls must be considered to participate in the work. In the shear wall and transformation tie-beam shear should be considered and vertical compressive stress, and considered as a combination.

\section{References}

[1] "JGJ138-2001", Technical Specification for Steel Reinforced Concrete Composite Structures, (2002).

[2] L. Wei, S. Wang, Journal of Building Structures, Vol.11(2001),pp7-14.

[3] B. Liang, F.S. Meng, etc., Journal of Henan University of Science and Technology (National Academy), Vol.25(2004),pp 68-73.

[4] S. Chen, F.S..Meng, etc. Journal of Tunnel Construction, Vol.23(2003),pp12-14.

[5] X.L. Sun etc. Journal of Engineering Construction and Design, Vol.5(2015),pp28-29. 